\title{
Progenitors of Long Gamma-ray Bursts
}

\author{
Melvyn B. Davies*, Andrew J. Levan ${ }^{\dagger, *}$, Josefin Larsson ${ }^{* * *}$, Andrew R. \\ King ${ }^{\ddagger}$ and Andrew S. Fruchter ${ }^{\S}$ \\ ${ }^{*}$ Lund Observatory, Box 43, SE-221 00 Lund, Sweden \\ ${ }^{\dagger}$ Department of Physics, University of Warwick, Coventry, CV4 7AL \\ ${ }^{* *}$ Institute of Astronomy, Madingley Road, Cambridge CB3 OHA, UK \\ ${ }^{\ddagger}$ Department of Physics and Astronomy, University of Leicester, Leicester LE1 7RH, UK \\ ${ }^{\S}$ Space Telescope Science Institute, 3700 San Martin Dr., Baltimore, MD 21218, USA
}

\begin{abstract}
Pinpointing the progenitors of long duration gamma-ray bursts (LGRBs) remains an extremely important question, although it is now clear that at least a fraction of LGRBs originate in the core collapse of massive stars in type Ic supernovae, the pathways to the production of these stars, and their initial masses, remain uncertain. Rotation is thought to be vital in the creation of LGRBs, and it is likely that black hole creation is also necessary. We suggest that these two constraints can be met if the GRB progenitors are very massive stars $>20 \mathrm{M}_{\odot}$ and are formed in tight binary systems. Using simple models we compare the predictions of this scenario with observations and find that the location of GRBs on their host galaxies are suggestive of mainsequence masses in excess of $20 \mathrm{M}_{\odot}$, while $50 \%$ of the known compact binary systems may have been sufficiently close to have had the necessary rotation rates for GRB creation. Thus, massive stars in compact binaries are a likely channel for at least some fraction of LGRBs.
\end{abstract}

Keywords: Gamma-ray bursts, Supernovae, Neutron Stars, Black holes

PACS: 98.70.Rz, 97.60.Bw, 97.60.Jd, 97.60.Lf

\section{INTRODUCTION}

The link between some long-duration gamma-ray bursts (LGRBs) and stellar collapse is now firmly established $[1,2,3]$ In particular LGRBs appear to originate in type Ic supernovae and frequently in hypernovae. There is significant diversity in the LGRB population, including the highly luminous ("classical") burst population, originating from a mean redshift of 2.8 [4], a nearby lower luminosity sub-class, which has a much higher space density (e.g. [5]), and possibly some LGRBs which are not associated with supernovae. This suggests there may be some variety in the progenitors of the bursts, and that they may not come from systems undergoing identical evolution. Nonetheless, there remains a strong drive to identify the progenitor stars themselves.

In the standard picture, core-collapse supernovae lead to LGRBs when the stellar core has a rapid rotation just prior to collapse. Some of the infalling material then forms a torus around the newly-formed central compact object (often assumed to be a black hole) and subsequent accretion of the material in the torus then fuels the LGRB. We are naturally left with two questions: 1) What leads to the high rotation rates required to form a torus? 2) What is the minimum progenitor mass required (or, in other words is the formation of a black hole (BH) rather than a neutron star (NS) necessary)?

For massive single stars, it is not clear whether sufficiently high central rotation rates may be maintained to produce a torus on core collapse (e.g. $[6,7])$, although it 
has been suggested that rapidly rotating metal-poor stars can retain sufficient angular momentum $[8,9]$ Alternatively, binary scenarios suggest a way of removing the envelope and providing a source of angular momentum (e.g. [10, 11]). We have explored this idea further [12] and present a summary of the key results here.

In addition, we have explored whether the observation of Fruchter et al. [13] that LGRBs are more concentrated on their host galaxy light than SNe may be due to the greater mass of a LGRB progenitor than a typical SNe progenitor [14]. A review of the key results of this work is also presented here.

\section{NEUTRON STAR BINARIES AND LONG-DURATION GAMMA-RAY BURSTS}

\section{Evolutionary pathways to compact object binaries}

Binaries containing two compact objects (ie white dwarfs, neutron stars or black holes) can be formed through several channels (see e.g. [15]). The basic scheme is shown in Fig. 1, and is essentially the same as that described in Bhattacharya and van den Heuvel [16].

The primary evolves first to produce either a black hole or neutron star, possibly following a phase of mass transfer to the secondary. When the secondary evolves, and fills its Roche lobe, the formation of a common envelope phase is likely owing to the mass ratio of the system. The black hole/neutron star and the helium-star core of the secondary will then spiral towards each other as the enshrouding gaseos envelope is ejected. The post-common envelope system will be a compact binary. A key point is that the helium-star core will be rotating rapidly because of tidal locking. In at least some systems, the rotation rate will be sufficiently large that when the secondary explodes as a core-collapse supernova, some of the infalling material will form a torus around the newly-formed neutron star or black hole.

\section{Discs around neutron stars and black holes}

In a core-collapse supernova, the material within the stellar core collapses to form a black hole or neutron star. If a core of a massive star is rotating sufficiently rapidly, some of the infalling material will be centrifugally supported (as it will carry its angular momentum with it) and form a torus around the central black hole or neutron star. The torus will have a radius of $D G M_{\mathrm{c}} / c^{2}$ (where $M_{\mathrm{c}}$ is the mass of the black hole or neutron star) if the specific angular momentum of the infalling material is given by $\sqrt{D} G M_{\mathrm{c}} / c$. In order to have a stable orbit around a (non-rotating) black hole, we require $D \geq 6$ (or a radius of around $12 \mathrm{~km}$ for a $1.4 M_{\odot}$ black hole). Forming a disc around a proto-neutron star requires more angular momentum as a disc would have to be formed with a radius somewhat larger than $10 \mathrm{~km}$.

If we assume that tidal locking occurs at the beginning of the helium main sequence and that for the late stages of evolution of the helium star the core decouples from the 
1)

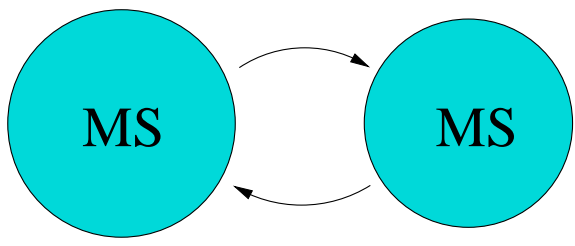

2)

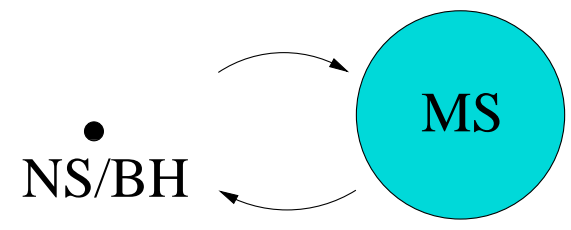

3)

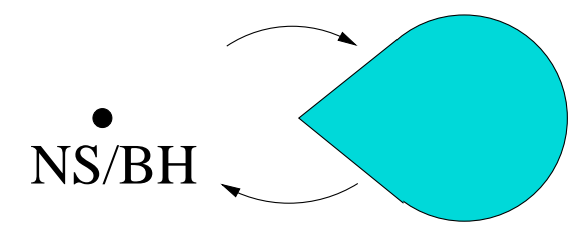

4)

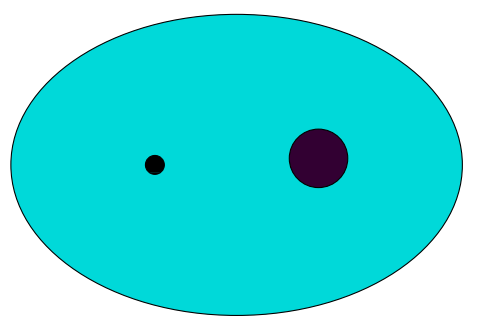

5)

6)

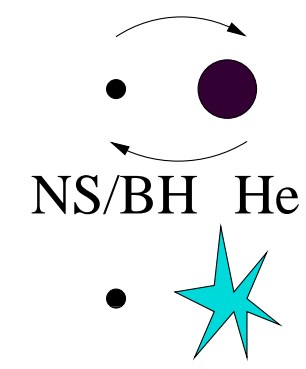

\section{$\mathrm{SN} / \mathrm{GRB}$}

FIGURE 1. An evolutionary pathway to the creation of a binary containing a rapidly-rotating corecollapse supernova in a tight orbit. The primary evolves first, possibly transferring material to the secondary (stage 1). It then produces a neutron star (NS) or black hole (BH), when it explodes as a corecollapse supernova (stage 2). The secondary then evolves, filling its Roche lobe (stage 3 ) and transferring material to the NS/BH producing a common envelope phase (stage 4). The NS/BH and He core of the secondary spiral together ejecting the surrounding envelope producing a very compact binary (stage 5). Tidal locking produces a rapidly-rotating $\mathrm{He}$ star such that the rotation is significant when the secondary explodes as a core-collapse supernova, with a torus being formed around the central compact object by infalling material. 
envelope. In this case, following Podsiadlowski et al. [11] we can compare the required angular momentum at the time of collapse with that at the edge of the iron core at the start of the helium main sequence, and, assuming tidal locking equate this to an orbital frequency (i.e. $\omega=\sqrt{D} G M_{\mathrm{c}} / R_{\mathrm{c}}^{2} c$, where $R_{\mathrm{c}}$ is the radius of the iron core). Assuming synchronous rotation, this gives a critical orbital separation [12]

$$
a<\left(4 M_{\mathrm{tot}} c^{2} R_{\mathrm{c}}^{4} / 9 D G M_{\mathrm{c}}^{2}\right)^{1 / 3}
$$

or

$$
\left(\frac{a}{R_{\odot}}\right)<\frac{60}{D^{1 / 3}}\left(\frac{R_{\mathrm{c}}}{R_{\odot}}\right)^{4 / 3}\left(\frac{M_{\mathrm{tot}}}{M_{\odot}}\right)^{1 / 3}\left(\frac{M_{\mathrm{c}}}{M_{\odot}}\right)^{-2 / 3}
$$

where $M_{\mathrm{c}}$ and $R_{\mathrm{c}}$ are the core mass and radius, and $M_{\text {tot }}$ is the total mass of the binary. We use here the models of Heger et al. [17], the key parameters are $M_{\mathrm{c}}=1.7 \mathrm{M}_{\odot}$, $R_{\mathrm{c}} \sim 0.1 R_{\odot}$, for a He star of mass $7.71 \mathrm{M}_{\odot}$, this yields a critical separation of $\sim 3 \mathrm{R}_{\odot}$ for the formation of a disc at the innermost stable orbit of a $1.7 M_{\odot} \mathrm{BH}$.

Do systems exist which are sufficiently tight to form discs when the secondary undergoes a core-collapse supernova? In order to answer this question, one can consider the observed compact binaries containing black holes or neutron stars which would be formed after the second supernova explosion (assuming the binary remained bound). There are currently no known black hole-neutron star binaries. However there are currently eight NS-NS binaries and three WD-NS binaries. The semi-major axis and eccentricity of a binary provides limits to the separation of the binary at the moment of the second supernova. In Fig. 2 we plot the semi-major axes and eccentricities of the observed systems. The plot also illustrates the minimum separation required to form a disc around the second compact object for discs of various radii. From this figure, we can see that discs are likely to have formed during the second supernova in about half of all osberved binaries. Energy released from such discs may power some of the long gamma-ray bursts, as discussed below.

\section{The contribution of compact binaries to long gamma-ray bursts}

Long-duration gamma-ray bursts are commonly thought to originate from discs around black holes located at the centre of a supernova. However, one can also consider whether discs around newly-formed neutron stars may also produce some form of gamma-ray burst. The maximum energy released in the accretion of the torus is given by $E=G M_{\mathrm{ns} / \mathrm{bh}} M_{\mathrm{acc}} / R_{\mathrm{ns} / \mathrm{bh}}$, or $E_{\mathrm{ns}}=3.6 \times 10^{53}\left(M_{\mathrm{acc}} / M_{\odot}\right)$ ergs, for a $1.4 \mathrm{M}_{\odot} \mathrm{NS}$ and $E_{\mathrm{bh}}=1 \times 10^{54}\left(M_{\mathrm{acc}} / M_{\odot}\right)$ ergs, for any mass BH (since $\left.M_{\mathrm{bh}} \propto R_{\mathrm{bh}}\right)$.

The extrapolation from disc accretion to gamma-ray luminosity is far from trivial since it requires an assumption about the conversion of accretion luminosity into $\gamma$-ray energy. One plausible mechanism of providing this energy is via neutrino-antineutrino annihilation. If this is assumed to be the energy source then the accretion energy can be related to the observed gamma-ray energy via several efficiency factors which account for the conversion of accretion energy to neutrinos, the cross section for neutrino antineutrino annihilation, the subsequent fraction of energy that is transferred into a 


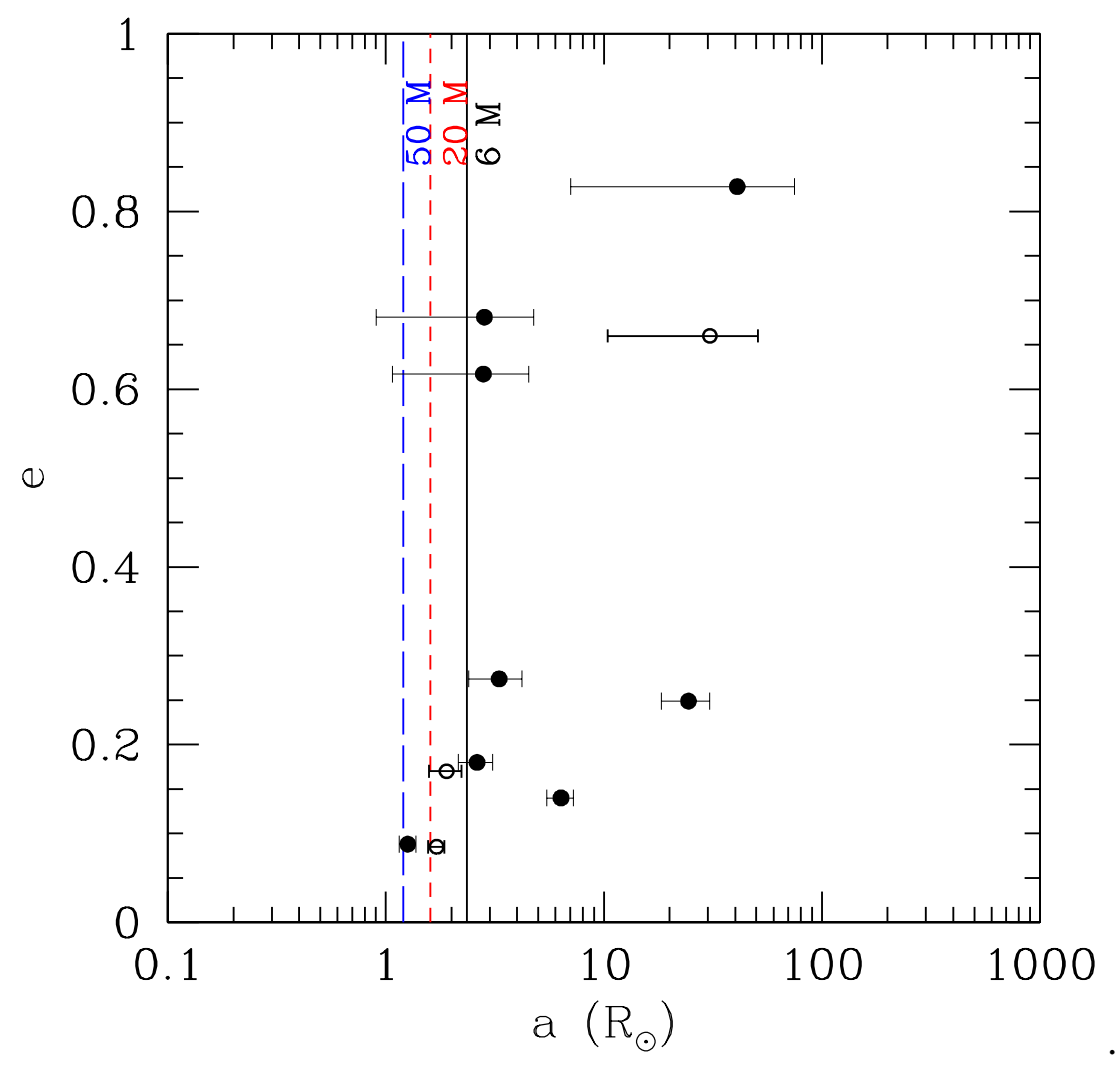

FIGURE 2. The eccentricity and semi-major axis of observed systems containing either two neutron stars, or a neutron star and white dwarf. The error bars give an indication of the range of separations between the two stars during their orbit. Neglecting the inspiral the separation at the time of the supernova must be taken from this range (see Table 1 for the calculations including the effects of inspiral via gravitational radiation). The three vertical lines represent the critical separations necessary at the time of core collapse for a centrifugally supported disc to have formed at a distance of 6M, 20M and 50M from the newly formed compact object, based on the models of Heger et al. [17] described in section 3 (where $M=G M_{n s / b h} / c^{2}$ and is $\sim 12,40$ and $100 \mathrm{~km}$ for $\mathrm{D}=6,20$ and 50 for a $\left.1.4 \mathrm{M}_{\odot} \mathrm{NS}\right)$. Lower total masses, as were likely the case of J0737-3039 require slightly tighter orbits, although the orbit is only a weak function of the total mass $\left(M_{t o t}^{1 / 3}\right)$. If the binary separation is less than this (i.e. to the left of the line) then disc formation is favoured. The data are from Champion et al. [18] and Lorimer [19]. In cases where the masses of the two components have not be measured 1.4 $\mathrm{M}_{\odot}$ has been assumed. Known NS-NS binaries are indicated with circles, while NS-WD or those with uncertain companions are marked with open circles

baryon free jet, and finally the fraction of this energy which is emitted as gammarays [20]. Following Oechslin and Janka [20] we assume that the product of the these efficiencies is $\sim 10^{-3}$. Thus the observed luminosities of low luminosity GRBs of $10^{48}-10^{50}$ ergs can be explained by the accretion of $0.01<\left(M_{a c c} / M_{\odot}\right)<0.3$ of material from the disc.

More-massive versions of the systems which formed the observed NS-NS binaries are expected to form $\mathrm{BH}-\mathrm{BH}$ binaries and are thus candidates for classical long gamma-ray bursts. Could both supernovae in such systems produce a gamma-ray burst? One can consider the separation required in order to give the core of the primary a sufficiently- 
high rotation rate. It is found that the size of the stellar is restricted to less than a few solar radii. In other words, the primary of a binary containing two massive stars is unlikely to be tidally spun-up sufficiently to produce a gamma-ray burst. However the black-hole it produces will work effectively to tidally spin-up the core of the secondary star following a pathway similar to that shown in Fig. 1.

\section{A NEW CONSTRAINT FOR GAMMA-RAY BURST PROGENITOR MASS}

\section{The basic idea}

Recently Fruchter et al. [13] have conducted a survey of the galactic environments of both long duration GRBs and core collapse SNe (i.e. all types of core collapse events, including SN II, Ib and Ic). These results demonstrate that GRBs are highly concentrated on their host light, significantly more so than the core collapse supernova population. Fruchter et al. [13] further suggest that this can be explained as being due to the GRBs originating in the most massive stars, which, upon core collapse form black holes rather than neutron stars. Here we further explore this possibility and attempt to derive plausible limits on the progenitor lifetime and mass based on the observed distributions of core collapse SNe and GRBs upon their host light. Using a simple model, motivated by the distributions of young star clusters in a local starburst galaxy, we explore the expected distributions of stars of different masses upon their host galaxies and compare these to the observed distributions from Fruchter et al. [13]. Our results demonstrate that for plausible models more massive stars are always more concentrated on their host light than lower mass stars. Further, given that supernovae originate from stars with initial masses $>8 \mathrm{M}_{\odot}$, we find that the observed distributions of GRBs on their host galaxies can naturally be explained by progenitors with initial masses in excess of 20 $\mathbf{M}_{\odot} \cdot$

\section{The model}

GRB host galaxies at high redshift are typically starburst galaxies. The Antennae (NGC 4038/4039) are a natural, local, analogue and were used as a template for constructing a simple model for GRB host galaxies. In this model we consider the population of young stellar clusters, as well as the stellar population contributing to the background light within the galaxy. The galaxy model consists of several key parameters: the surface density of clusters; the distribution of cluster masses; the distribution of cluster ages; the distribution of background light; and the distribution of clusters on the background light. The first two properties were taken from observations of NGC 4038/4039 when viewed at $z \sim 1$. The surface density of clusters expressed in terms of number of clusters per pixel is $\sim 0.15$, although this is far from uniform across the galaxy. We

use the observed distribution of cluster masses, which follows $d N / d M_{\mathrm{cl}} \propto M_{\mathrm{cl}}^{-2}$, with $M_{\mathrm{cl} \text {,min }}=4 \cdot 10^{4} M_{\odot}$ and $M_{\mathrm{cl}, \max }=10^{6} M_{\odot}$, where $M_{\mathrm{cl}}$ is the cluster mass. The age dis- 


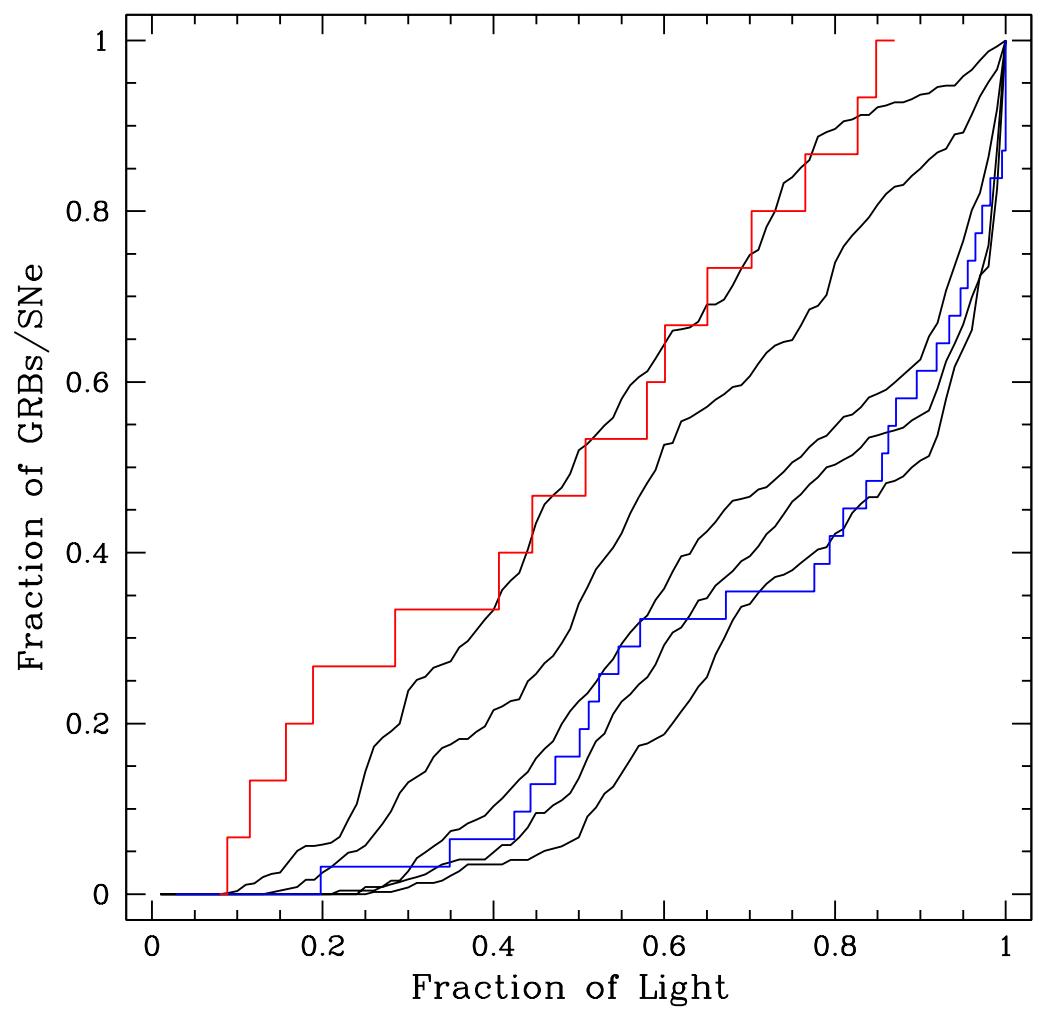

FIGURE 3. Fraction of objects plotted against fraction of light for observed $\mathrm{SNe}$ (red) and GRBs (blue) together with the results from our model (black lines). Black lines from top to bottom correspond to minimum progenitor masses of $8,20,40,60$, and $80 M_{\odot}$.

tribution of clusters was taken from Fall et al. [21] and follows $d N / d \tau \propto \tau^{-1}$, where $\tau$ is the cluster age. In order to mimic an (almost) instantaneous burst of star formation, clusters are created in the model according to this distribution over a period of $10^{7}$ years. The distribution of the background light adopted lies in the middle of the distributions observed for galaxies hosting supernovae and gamma-ray bursts and is given by $d N / d L_{\text {pix }} \propto L_{\text {pix }}^{-1.5}$ with $L_{\text {pix,max }} / L_{\text {pix,min }}=20$, where $L_{\text {pix }}$ is the luminosity of a pixel. We distribute the stellar clusters such that there is a correlation between background brightness and the number of clusters present in a pixel. The degree of correlation was chosen to give a good match to the cluster distribution observed in NGC 4038/39.

\section{Results}

Using the parameters described in the previous section we performed runs for minimum progenitor masses of $8,20,40,60$, and $80 M_{\odot}$. The results are shown as black lines in Fig. 3 together with the observed distributions of $\mathrm{SNe}$ (in red) and GRBs (in blue) from Fruchter et al. [13]. 


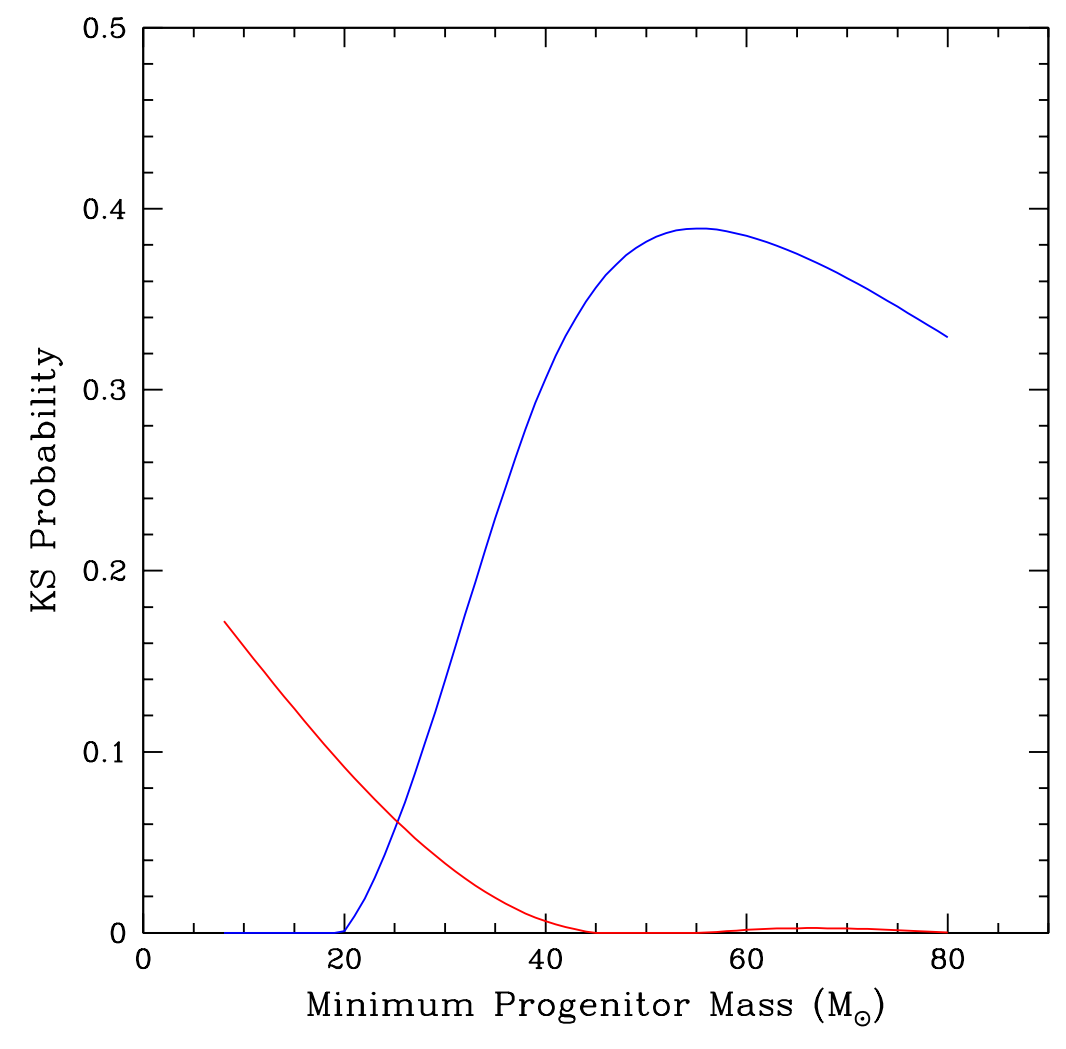

FIGURE 4. KS-probabilities of our model results following the observed SN (red) and GRB (blue) distributions. The probabilities are plotted as a function of the minimum progenitor mass and were calculated for masses of $8,20,40,60$, and $80 M_{\odot}$. A spline has been fitted through the data points.

The model distributions for all masses were KS-tested against the observed SN and GRB distributions and the resulting probabilities are shown as a function of mass in Fig. 4. While the probability of following the SN distribution decreases with increasing mass, the likelihood of following the observed GRB distribution increases rapidly from 8 to 40 $M_{\odot}$ and then flattens out, reaching a weak maximum around $60 M_{\odot}$. The shapes of the two probability functions look the same for all realisations of the model, although the peak probabilities can change by about 0.1 between different runs. These results strongly suggest that GRB progenitors are significantly more massive than SN progenitors.

\section{SUMMARY}

We have suggested that GRBs originate from the most massive stars [14] and that many of these stars exist within tight binary systems [12]. After the GRB a NS-NS, NS-BH or BH-BH binary may remain, as the separation at the time of the GRB is moderately small many of the systems will merge within a Hubble time and might be observable as a short GRB. These systems are spectacular, at some point in their evolution they will be observed as two supernova, two GRBs as well as X-ray binary (and possibly even 
ultraluminous X-ray source) phases.

We note that while it is likely that LGRBs are formed via this channel it is not necessarily the only one operating, indeed the diversity in the properties of observed GRBs implies there may be significant variations in the progenitors themselves, for example the low luminosity burst population may be formed via neutron star creating supernovae $[12,22]$ while single stars, which undergo complete mixing on the main sequence (e.g. [9]) may also create a subset of the GRBs.

\section{ACKNOWLEDGMENTS}

MBD is a Royal Swedish Academy Research Fellow supported by a grant from the Knut and Alice Wallenberg Foundation. AJL is grateful to PPARC for a postdoctoral fellowship award. AJL also thanks the Swedish Institute for support while visiting Lund.

\section{REFERENCES}

1. S. E. Woosley, ApJ 405, 273-277 (1993).

2. J. Hjorth, J. Sollerman, P. Møller, J. P. U. Fynbo, S. E. Woosley, C. Kouveliotou, N. R. Tanvir, J. Greiner, M. I. Andersen, A. J. Castro-Tirado, J. M. Castro Cerón, A. S. Fruchter, J. Gorosabel, P. Jakobsson, L. Kaper, S. Klose, N. Masetti, H. Pedersen, K. Pedersen, E. Pian, E. Palazzi, J. E. Rhoads, E. Rol, E. P. J. van den Heuvel, P. M. Vreeswijk, D. Watson, and R. A. M. J. Wijers, Nature 423, 847-850 (2003), astro-ph/0306347.

3. K. Z. Stanek, T. Matheson, P. M. Garnavich, P. Martini, P. Berlind, N. Caldwell, P. Challis, W. R. Brown, R. Schild, K. Krisciunas, M. L. Calkins, J. C. Lee, N. Hathi, R. A. Jansen, R. Windhorst, L. Echevarria, D. J. Eisenstein, B. Pindor, E. W. Olszewski, P. Harding, S. T. Holland, and D. Bersier, ApJ 591, L17-L20 (2003), astro-ph/0304173.

4. P. Jakobsson, A. Levan, J. P. U. Fynbo, R. Priddey, J. Hjorth, N. Tanvir, D. Watson, B. L. Jensen, J. Sollerman, P. Natarajan, J. Gorosabel, J. M. Castro Cerón, K. Pedersen, T. Pursimo, A. S. Árnadóttir, A. J. Castro-Tirado, C. J. Davis, H. J. Deeg, D. A. Fiuza, S. Mykolaitis, and S. G. Sousa, $A \& A$ 447, 897-903 (2006), astro-ph/0509888.

5. E. Pian, P. A. Mazzali, N. Masetti, P. Ferrero, S. Klose, E. Palazzi, E. Ramirez-Ruiz, S. E. Woosley, C. Kouveliotou, J. Deng, A. V. Filippenko, R. J. Foley, J. P. U. Fynbo, D. A. Kann, W. Li, J. Hjorth, K. Nomoto, F. Patat, D. N. Sauer, J. Sollerman, P. M. Vreeswijk, E. W. Guenther, A. Levan, P. O’Brien, N. R. Tanvir, R. A. M. J. Wijers, C. Dumas, O. Hainaut, D. S. Wong, D. Baade, L. Wang, L. Amati, E. Cappellaro, A. J. Castro-Tirado, S. Ellison, F. Frontera, A. S. Fruchter, J. Greiner, K. Kawabata, C. Ledoux, K. Maeda, P. Møller, L. Nicastro, E. Rol, and R. Starling, Nature 442, 1011-1013 (2006), astro-ph/0603530.

6. A. I. MacFadyen, and S. E. Woosley, ApJ 524, 262-289 (1999), astro-ph/9810274.

7. J. Petrovic, N. Langer, and K. A. van der Hucht, A\&A 435, 1013-1030 (2005), astro-ph/0504242.

8. S.-C. Yoon, and N. Langer, $A \& A$ 443, 643-648 (2005), astro-ph/0508242.

9. S. E. Woosley, and A. Heger, ApJ 637, 914-921 (2006), astro-ph/0508175.

10. R. G. Izzard, E. Ramirez-Ruiz, and C. A. Tout, MNRAS 348, 1215-1228 (2004), astro-ph/0311463.

11. P. Podsiadlowski, P. A. Mazzali, K. Nomoto, D. Lazzati, and E. Cappellaro, ApJ 607, L17-L20 (2004), astro-ph/0403399.

12. A. J. Levan, M. B. Davies, and A. R. King, MNRAS 372, 1351-1356 (2006), astro-ph/0608316.

13. A. S. Fruchter, A. J. Levan, L. Strolger, P. M. Vreeswijk, S. E. Thorsett, D. Bersier, I. Burud, J. M. Castro Cerón, A. J. Castro-Tirado, C. Conselice, T. Dahlen, H. C. Ferguson, J. P. U. Fynbo, P. M. Garnavich, R. A. Gibbons, J. Gorosabel, T. R. Gull, J. Hjorth, S. T. Holland, C. Kouveliotou, Z. Levay, M. Livio, M. R. Metzger, P. E. Nugent, L. Petro, E. Pian, J. E. Rhoads, A. G. Riess, K. C. Sahu, A. Smette, N. R. Tanvir, R. A. M. J. Wijers, and S. E. Woosley, Nature 441, 463-468 (2006), astro-ph/0603537. 
14. J. Larsson, A. J. Levan, M. B. Davies, and A. S. Fruchter, MNRAS (2007), accepted, astro$\mathrm{ph} / 0701562$.

15. K. Belczynski, V. Kalogera, and T. Bulik, ApJ 572, 407-431 (2002), astro-ph/0111452.

16. D. Bhattacharya, and E. P. J. van den Heuvel, Phys. Rep. 203, 1-124 (1991).

17. A. Heger, N. Langer, and S. E. Woosley, ApJ 528, 368-396 (2000), astro-ph/9904132.

18. D. J. Champion, D. R. Lorimer, M. A. McLaughlin, J. M. Cordes, Z. Arzoumanian, J. M. Weisberg, and J. H. Taylor, MNRAS 350, L61-L65 (2004), astro-ph/0403553.

19. D. R. Lorimer, Living Reviews in Relativity 8, 7-+ (2005), astro-ph/0511258.

20. R. Oechslin, and H.-T. Janka, MNRAS 368, 1489-1499 (2006), astro-ph/0507099.

21. S. M. Fall, R. Chandar, and B. C. Whitmore, ApJ 631, L133-L136 (2005), astro-ph/0509293.

22. A. M. Soderberg, S. R. Kulkarni, E. Nakar, E. Berger, P. B. Cameron, D. B. Fox, D. Frail, A. GalYam, R. Sari, S. B. Cenko, M. Kasliwal, R. A. Chevalier, T. Piran, P. A. Price, B. P. Schmidt, G. Pooley, D.-S. Moon, B. E. Penprase, E. Ofek, A. Rau, N. Gehrels, J. A. Nousek, D. N. Burrows, S. E. Persson, and P. J. McCarthy, Nature 442, 1014-1017 (2006), astro-ph/0604389. 